\title{
Cardiovascular secondary prevention in high-risk patients: a randomized controlled trial sub-study
}

\author{
Stina Jakobsson ${ }^{*}$, Anna-Lotta Irewall, Fredrik Bjorklund and Thomas Mooe
}

\begin{abstract}
Background: Enhanced cardiovascular secondary preventive follow-up is needed to improve adherence to recommended low-density lipoprotein cholesterol (LDL-C) and blood pressure (BP) levels. Patients with diabetes mellitus (DM) or chronic kidney disease (CKD) have a high risk of recurrent events. Secondary prevention is therefore essential in these patients.

Methods: Patients with acute coronary syndrome, stroke, or transient ischemic attack were randomized to nurse-based telephone follow-up (intervention) or usual care (control). LDL-C and BP were measured at 1 month (baseline) and 12 months post-discharge. Intervention patients with above-target values at baseline received medication titration to achieve treatment goals. Values measured for control patients were given to the patient's general practitioner for assessment.

Results: The final analyses included 225 intervention and 215 control patients with DM or CKD. Among patients with above-target baseline values, the following 12-month values were recorded for intervention and control patients, respectively: $L D L-C, 2.2$ versus $3.0 \mathrm{mmol} / \mathrm{L}(p<0.001)$; and median systolic $B P(S B P), 140$ versus $145 \mathrm{mmHg}(p=0.26)$. Among patients with above-target values at baseline, $52.3 \%$ of intervention patients reached target $L D L-C$ values at 12 months versus $21.3 \%$ of control patients (absolute difference of $30.9 \%, 95 \% \mathrm{Cl} 16.1$ to $43.8 \%$ ), and there was a non-significant trend of more intervention patients reaching target SBP ( $49.4 \%$ versus $36.8 \%$; absolute difference of $12.6 \%, 95 \% \mathrm{Cl}-1.7$ to $26.2 \%)$.
\end{abstract}

Conclusions: Cardiovascular secondary prevention with nurse-based telephone follow-up was more effective than usual care in improving LDL-C levels 12 months after discharge for patients with DM or CKD.

Trial registration: ISRCTN registry; ISRCTN96595458 (date of registration 10 July 2011) and ISRCTN23868518 (date of registration 13 May 2012).

Keywords: Acute coronary syndrome, Myocardial infarction, Stroke, Transient ischemic attack, Diabetes mellitus, Chronic renal insufficiency, Secondary prevention, Cardiovascular disease

\section{Background}

Following an acute coronary syndrome (ACS) or stroke there is a high risk of recurrent events. A population based study of patients with ischemic stroke found that the cumulative five year risk of MI, stroke or vascular event was 29 \% [1]. Furthermore, data from Swedish Web-system for Enhancement and Development of Evidence-based care in Heart disease Evaluated According to Recommended

\footnotetext{
* Correspondence: stina.jakobsson@umu.se

Department of Public Health and Clinical Medicine, Division of Medicine, Ostersund sjukhus, Umea University, Umea, Sweden
}

Therapies (SWEDEHEART) show that $13 \%$ of the patients are readmitted for a cardiac disease during the year following a myocardial infarction and $4 \%$ suffer an ischemic stroke within one year [2,3]. Morbidity and case fatality after an ACS or stroke can be reduced by secondary preventive treatments that lower lipid levels and blood pressure (BP) [4]. However, implementation of such treatments has been unexpectedly difficult, with low proportions of patients reaching the recommended blood lipid levels and BP [5-7]. In EUROASPIRE IV patients with an ACS or who were revascularized with percutaneous coronary 
intervention or coronary artery bypass grafting were examined between six months and three years after the qualifying event. Approximately $80-90 \%$ of the patients received a blood pressure lowering drug and a statin, but of those receiving treatment only 57 and $21 \%$ reached target levels for BP and low-density lipoprotein cholesterol (LDL-C), respectively [7]. Various organizational strategies have been evaluated for improving secondary preventive care in patients with established cardiovascular disease (CVD), including patient education, physician education, prebooked doctor appointments, etc. However, most investigated strategies have not effectively reduced risk factors $[8,9]$. Some data suggest that telephone-based follow-up improves risk factor control [10].

Diabetes mellitus (DM) and chronic kidney disease (CKD) are common comorbidities in patients with established CVD. In a population of Medicare beneficiaries of 65 years of age or older in the United States, DM was found in approximately $40 \%$ of the patients with ischemic heart disease or stroke and CKD was found in 30-35\% of the patients [11]. Moreover, patients with DM or CKD have a worse prognosis after a CVD. For example, one study showed that the hazard ratio of death for DM patients compared to non-DM patients one year after an ACS was increased (1.33; $95 \%$ confidence interval 1.20-1.48) and a pooled analysis of community-based cohorts showed that over $60 \%$ of the patients with established CVD and CKD suffered a MI, fatal coronary heart disease, stroke, or died during a 10-year time period, compared to approximately $35 \%$ of the non-CKD patients [12, 13]. While the agestandardized death rates after an ACS or stroke have decreased over recent years [14], the prevalences of DM and CKD are increasing $[15,16]$. Thus, there is a potentially growing population with CVD in combination with DM and/or CKD. These patients have a very high risk of recurrent events and secondary prevention is therefore essential.

The Nurse-Based Age-Independent Intervention to Limit Evolution of Disease (NAILED) ACS and NAILED stroke risk factor trials are ongoing randomized controlled trials that aim to improve secondary prevention after ACS, stroke, and transient ischemic attack (TIA) through nurse-based telephone follow-up of modifiable risk factors. Considering the importance of secondary prevention, we performed the present study to investigate the effects of follow-up according to the NAILED trial in a high-risk patient population with DM or CKD. We hypothesized that compared to usual care, the NAILED follow-up would more effectively improve LDL-C levels and BP at 12 months after an ACS, stroke, or TIA.

\section{Method}

The rationale and the open, population-based, randomized controlled design of the NAILED trials have been previously described $[17,18]$. In brief, all patients hospitalized at Ostersund Hospital with a diagnosis of myocardial infarction, unstable angina, stroke, or TIA were assessed for inclusion. Ostersund Hospital is the only hospital in the county of Jamtland, Sweden, and has a rural catchment area with a population of approximately 125,000 inhabitants. Study nurses identified eligible patients with the physical and mental capacity to communicate by telephone. Patients suffering from deafness, aphasia, dementia, or severe (often terminal) diseases were not included. Participants in other ongoing trials were also excluded. All eligible patients were informed about the study and asked to sign a written informed consent. Those who agreed to participate were randomized to intervention or control in a 1:1 ratio. The random allocation sequence was computer generated in blocks of four and stratified for sex and type of ACS (unstable angina or myocardial infarction) for ACS patients. For patients with stroke or TIA the random sequence was generated in blocks of four and was stratified for sex and for degree of disability (modified Rankin Scale $<3$ or $\geq 3$ ).

This study was approved by the Regional Ethics Committee, Umea University 28 October 2009 (reference number Dnr-09-142M).

Patient characteristics, including medical history, were recorded during the initial hospitalization. At 1 month after discharge, baseline measurements of blood lipids and BP were performed by a health care professional at the patients' closest health care facility and reported to the study team. Corresponding follow-up measurements were performed at 12 months after discharge. Shortly after the measurements of blood lipids and BP, a study nurse contacted participants in both study groups by telephone and interviewed them about their well-being and adherence to medical treatment.

In the control group, LDL-C and BP values were forwarded directly to the patient's general practitioner (GP) for assessment, without further action from the study team. The patients in the control group received secondary preventive care according to local standard management. Secondary preventive treatment was generally initiated inhospital and thereafter, the patients' GPs held primary responsibility for their care. The standard of care for DM patients is, according to local guidelines, a yearly visit to their GP and a yearly visit to a nurse. During the visits a risk factor assessment and intervention regarding established risk factors for CVD is performed. The secondary preventive management of CKD patients does not differ from the overall CVD population. Patients in both study groups could at any time book an appointment with their GP.

For patients randomized to intervention, if they had not reached the target LDL-C level and/or BP their medication was titrated according to guidelines. The initial target value for LDL-C level was $<2.5 \mathrm{mmol} / \mathrm{L}$. On 31 March, 2013, the local guidelines for diabetic patients 
changed such that the target level was $<1.8 \mathrm{mmol} / \mathrm{L}$. Throughout the trial period, the target $\mathrm{BP}$ value was $<140 / 90 \mathrm{mmHg}$. Tests were repeated within approximately 4 weeks. If necessary, medication was further titrated until target values were reached or no further changes were considered appropriate. Decisions regarding titration and medication were made by a study physician.

The present analysis included patients with DM or CKD, who were admitted between 1 January, 2010 and 30 June, 2013 and who received a 12-month follow-up call. Patients were considered to have DM if they received glucose-lowering medication or dietary treatment at discharge from their initial hospitalization. During hospitalization, a creatinine value was registered and an estimated glomerular filtration rate (eGFR) was calculated using the Chronic Kidney Disease Epidemiology Collaboration (CKD-EPI) creatinine equations [19]. Patients were assumed to be Caucasian. An eGFR $<60 \mathrm{~mL} /$ $\mathrm{min} / 1.73 \mathrm{~m}^{2}$ was considered to indicate the presence of CKD [20].

\section{Outcomes}

The primary outcome in the present analysis was to determine the difference in LDL-C and systolic blood pressure (SBP) between patients in the two groups at 12 months follow-up, before any medication titrations. The differences in diastolic blood pressure (DBP) and proportions of reached target values between the groups were secondary outcomes. Exploratory data analyses with between-group comparison of risk factor values and proportion of patients within target values were performed separately for patients with DM and CKD.

\section{Availability of data}

Deposition of patient level data in a public repository was not specified in the study protocol as approved by the ethics committee before the study started. Patient level data will be available on request, provided that an approval from the Regional Ethics Committee is given.

\section{Statistics}

The results are presented as median and percentiles for continuous variables, and as percentages for categorical variables. To analyse the effects of the intervention, risk factor values and the proportion of achieved target values at 12 months were analysed separately among patients who did and did not meet risk factor target values at baseline. To assess between-group differences, the MannWhitney $U$-test was used for continuous variables and Pearson's $\chi^{2}$ test for categorical variables. Within-group changes from baseline to 12-month follow-up were examined using the Wilcoxon signed-rank test.
Power analysis showed that study groups of approximately 80 participants were required to detect betweengroup differences of $0.4 \mathrm{mmol} / \mathrm{L}$ in mean LDL-C, of $8 \mathrm{mmHg}$ in mean SBP, and of $20 \%$ in the proportions of reached target values (alpha 0.05, two-tailed, power $80 \%)$. Subjects with missing values were excluded from the analysis. The Bonferroni technique was used to adjust the alpha value because multiple comparisons were performed within each group. After adjustment an alpha value of 0.01 was considered significant in the risk factor value analyses. Statistical analyses were performed using SPSS 20.0 software.

\section{Results}

Between January 2010 and June 2013, 1511 ACS, stroke, or TIA patients were included in the study. Of these subjects, 758 (33.9 \% [257/758] with DM or CKD) were randomized to the intervention group and 753 (33.6\% [253/753] with DM or CKD) to the control group. The final analyses included 225 patients with DM or CKD in the intervention group and 215 patients in the control group (Fig. 1). Table 1 shows the patients' in-hospital characteristics. The groups were well matched except for that fewer patients in the intervention group received lipid-lowering treatment at admission (40.9\% versus $52.6 \%$ ). Thirty-five patients in the intervention group and 36 patients in the control group had both $\mathrm{DM}$ and CKD.

\section{Risk factor values at 12 months}

The baseline median values for LDL-C, SBP, and DBP, for patients not meeting target values were similar between the intervention and control groups (Table 2). At the 12-month follow-up, both groups showed significant decreases in these risk factor values compared to baseline. LDL-C levels at 12 months were significantly lower in the intervention group $(2.2 \mathrm{mmol} / \mathrm{L})$ compared to the control group $(3.0 \mathrm{mmol} / \mathrm{L})(p<0.001)$. We observed a non-significant trend towards a lower SBP value in the intervention group $(140 \mathrm{mmHg})$ compared to in the control group $(145 \mathrm{mmHg})(p=0.26)$ (Table 2). Additional file 1 shows the corresponding data for the patients who met target values at baseline. Among these patients, the risk factor values generally increased between baseline and 12 months and there were no significant between-group differences.

\section{Proportions of patients who achieved target values at 12 months}

At baseline, the target LDL-C level was met by $60.8 \%$ $(135 / 222)$ in the intervention group and by $62.7 \%(131 /$ 209) of the control group (absolute difference of $1.9 \%$, $95 \%$ CI -7.2 to $10.9 \%)$, target SBP by $60.3 \%(135 / 224)$ of the intervention group and $55.8 \%(120 / 215)$ of controls 


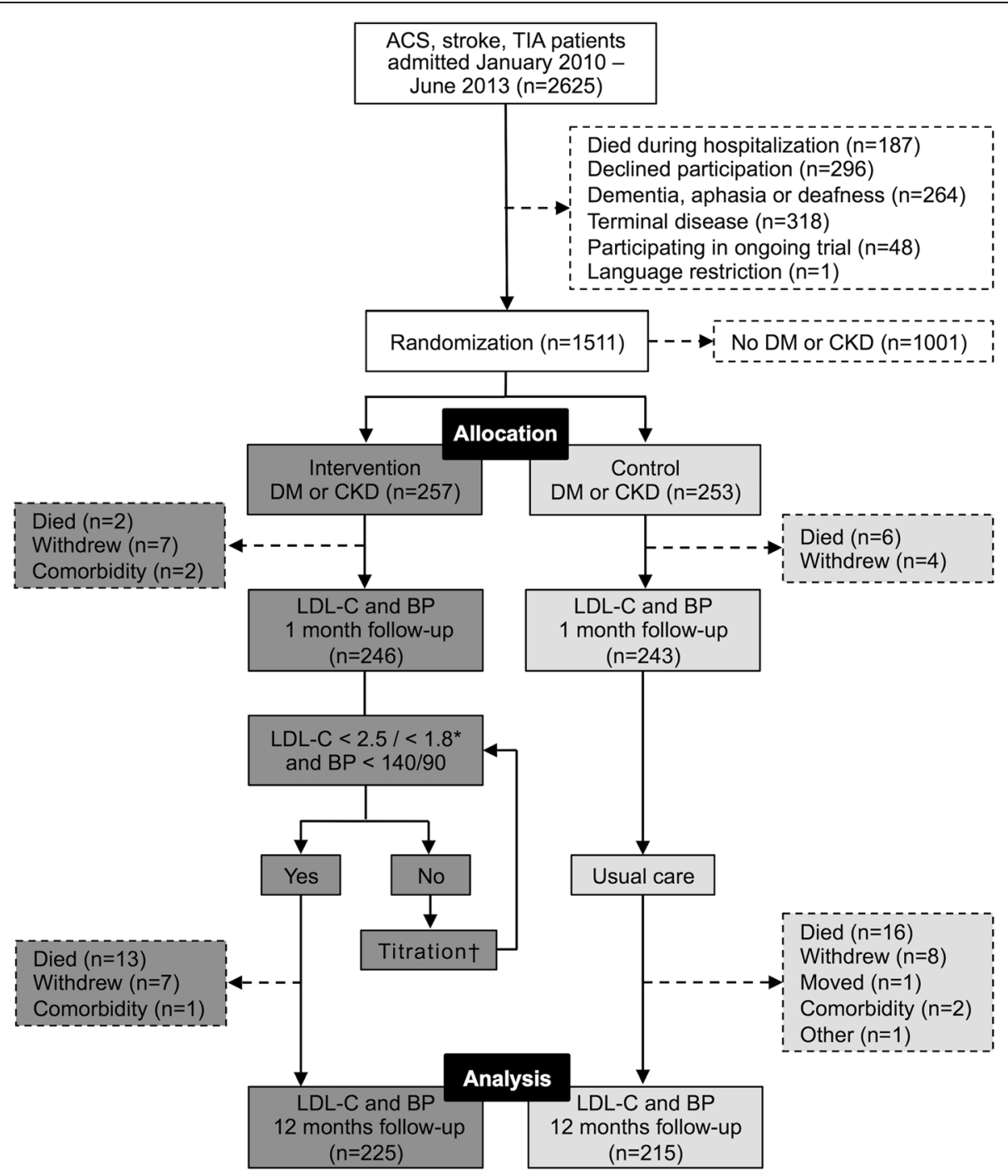

Fig. 1 Study flow diagram. *The target LDL-C value was <2.5 mmol/L until 31 March, 2013, when local guidelines for diabetic patients changed this target to $<1.8 \mathrm{mmol} / \mathrm{L}$; † Medication was titrated until target values were reached or no further changes were considered appropriate. $n$ : number of cases; DM: diabetes mellitus; CKD: chronic kidney disease; LDL-C: low-density lipoprotein cholesterol; BP: blood pressure

(absolute difference of $4.5 \%, 95 \%$ CI -4.7 to $13.6 \%$ ), and target DBP by $88.8 \%(199 / 224)$ of the intervention group and $87.9 \%(189 / 215)$ of controls (absolute difference of $0.9 \%, 95 \% \mathrm{CI}-5.1$ to $7.1 \%)$. Figure 2 shows the proportions of patients who reached target LDL-C and SBP values at 12 months stratified by baseline values (reaching target or not). Among patients with above-target values at baseline, a significantly higher proportion of the intervention group showed target LDL-C values at 12 months compared to controls (52.3\% [45/86] versus $21.3 \%$ [16/75]; absolute difference of $30.9 \%, 95 \%$ CI 16.1 to $43.8 \%$ ). The corresponding proportions of target SBP values at 12 months for patients with above target at baseline were $49.4 \%(44 / 89)$ and $36.8 \%$ (35/95) (absolute difference of $12.6 \%, 95 \% \mathrm{CI}-1.7$ to $26.2 \%$ ), respectively.
Figure 3 shows the proportion of patients who met all target values (LDL-C, SBP and DBP) at baseline, after medication titration, and at 12 months. At baseline this proportion did not differ between the groups (intervention $34.2 \%$ [76/222] versus control $35.1 \%$ [74/211]; absolute difference of $0.8 \%, 95 \%$ CI -8.1 to $9.8 \%)$. After medication titration, $83.8 \%(186 / 222)$ of the patients in the intervention group reached all target values (including patients who already showed target values at baseline) (absolute difference of $48.7 \%$, $95 \%$ CI 40.1 to $56.2 \%)$. At 12 months $40.0 \%(88 / 220)$ of the intervention patients and $28.2 \%(59 / 209)$ of the control patients met all target values (absolute difference of $11.8 \%$, $95 \%$ CI 2.8 to $20.5 \%)$. 
Table 1 In-hospital patient characteristics

\begin{tabular}{|c|c|c|}
\hline & & \\
\hline & $n=225$ & $n=215$ \\
\hline $\begin{array}{l}\text { Age in years, median (25th-75th } \\
\text { percentile) }\end{array}$ & $75(67-82)$ & $75(67-80)$ \\
\hline Female, $n(\%)$ & $86(38.2)$ & $75(34.9)$ \\
\hline Smoking, $n(\%)$ & $25(11.1)$ & $23(10.7)$ \\
\hline BMI, median (25th-75th percentile) & $27.9(24.7-31.0)$ & $27.2(24.6-30.2)$ \\
\hline Diabetes mellitus, $n$ (\%) & $139(61.8)$ & $128(59.5)$ \\
\hline Chronic kidney disease, $n$ (\%) & $121(53.8)$ & $123(57.2)$ \\
\hline \multicolumn{3}{|l|}{ Prior disease, $n(\%)$} \\
\hline Coronary artery disease & $52(23.1)$ & $57(26.5)$ \\
\hline Stroke/TIA & $52(23.1)$ & $44(20.5)$ \\
\hline Peripheral artery disease & $8(3.6)$ & $9(4.2)$ \\
\hline \multicolumn{3}{|l|}{ Qualifying event, $n(\%)$} \\
\hline Unstable angina & $7(3.1)$ & $9(4.2)$ \\
\hline NSTEMI & $82(36.4)$ & 70 (32.6) \\
\hline STEMI & $30(13.3)$ & $24(11.2)$ \\
\hline $\mathrm{TIA}$ & 35 (15.6) & $42(19.5)$ \\
\hline Ischemic stroke & $68(30.2)$ & $66(30.7)$ \\
\hline Haemorrhagic stroke & $3(1.3)$ & $4(1.9)$ \\
\hline \multicolumn{3}{|l|}{ Treatment at admission, $n(\%)$} \\
\hline Antihypertensive drug $(\geq 1)$ & $183(81.3)$ & $188(87.4)$ \\
\hline Lipid-lowering drug & $92(40.9)$ & $113(52.6)$ \\
\hline \multicolumn{3}{|l|}{ Treatment at discharge, $n(\%)$} \\
\hline Antihypertensive drug $(\geq 1)$ & $211(93.8)$ & $199(92.6)$ \\
\hline Lipid-lowering drug & $185(82.2)$ & $180(83.7)$ \\
\hline
\end{tabular}

$n$ number of cases, BMI body mass index (calculated as weight in kilograms divided by the squared height in meters), TIA transient ischemic attack, NSTEMI non-ST elevation myocardial infarction, STEMI ST elevation myocardial infarction

\section{Sub-group analysis}

Repeating the analyses separately for patients with DM and CKD produced results that were largely similar to the combined analyses with regard to risk factor value differences between intervention and control patients (see Additional files 2 and 3). The separate analyses showed that $43.5 \%(20 / 46)$ of DM patients in the intervention group whose LDL-C levels were above target at baseline had reached target LDL-C levels at 12 months, compared to $26.5 \%(9 / 34)$ in the control group (absolute difference of $17.0 \%, 95 \% \mathrm{CI}-4.3$ to $35.6 \%$ ). The corresponding results in the CKD group were $60.4 \%$ $(32 / 53)$ versus $14.3 \%(7 / 49)$, respectively (absolute difference of $46.1 \%, 95 \%$ CI 27.8 to $60.1 \%$ ).

Among the CKD patients in the intervention group who showed above-target SBP at baseline, separate analysis showed that $59.6 \%$ had reached target at 12 months, while combined DM and CKD analysis showed a lower proportion (49.4\%). Thus, more CKD intervention patients reached target SBP at 12 months compared to CKD control patients (59.6\% [28/47] versus $35.2 \%$ [19/54]; absolute difference of $24.3 \%$, $95 \%$ CI 4.9 to $41.5 \%$ ).

\section{Discussion}

The present results demonstrated that a structured, secondary preventive, nurse-based telephone follow-up of modifiable risk factors after an ACS, stroke, or TIA led to more prevalent LDL-C level improvement over 12 months among high-risk patients with DM or CKD as compared to the impact of standard care. We also observed a more prevalent decrease of SBP in the intervention group compared to the control group, but this difference was not statistically significant-possibly because the patient groups were too small. A betweengroup difference of $5 \mathrm{mmHg}$ is probably of clinical significance; however, the power of the study was only sufficient to detect an $8 \mathrm{mmHg}$ difference. With regard

Table 2 Risk factor values for patients who had above-target values at baseline

\begin{tabular}{llll}
\hline & Intervention & Control & $p$-value \\
\hline LDL-C $\geq 2.5 / 1.8^{a}$ baseline, $n$ & 87 & 78 & $2.9(2.7-3.7)$ \\
LDL-C baseline, median (25th-75th percentile) & $2.9(2.6-3.5)$ & $3.0(2.4-3.4)^{* *}$ \\
LDL-C 12 months, median (25th-75th percentile) & $2.2(1.8-3.0)^{* * *}$ & 95 & 0.74 \\
SBP $\geq 140$ baseline, $n$ & 89 & $150(140-160)$ \\
SBP baseline, median (25th-75th percentile) & $150(142-160)$ & $145(130-158)^{* * *}$ \\
SBP 12 months, median (25th-75th percentile) & $140(129-155)^{* * *}$ & 26 & 0.97 \\
DBP $\geq 90$ baseline, $n$ & 25 & $90(90-96)$ \\
DBP baseline, median (25th-75th percentile) & $90(90-95)$ & $80(75-91)^{* * *}$ \\
DBP 12 months, median (25th-75th percentile) & $80(75-86)^{* * *}$ & 0.76 \\
\hline
\end{tabular}

${ }^{* *} p \leq 0.01,{ }^{* * *} p \leq 0.001$, indicating a significant change of median values within each group between baseline and 12 months; ${ }^{\text {a }}$ The target $L D L-C$ value was $<2.5 \mathrm{mmol} / \mathrm{L}$ until $31 \mathrm{March}, 2013$, when local guidelines for diabetic patients changed this target to $<1.8 \mathrm{mmol} / \mathrm{L}$. LDL-C low-density lipoprotein cholesterol $(\mathrm{mmol} / \mathrm{L}), n$ number of cases, SBP systolic blood pressure $(\mathrm{mmHg}), D B P$ diastolic blood pressure $(\mathrm{mmHg})$ 


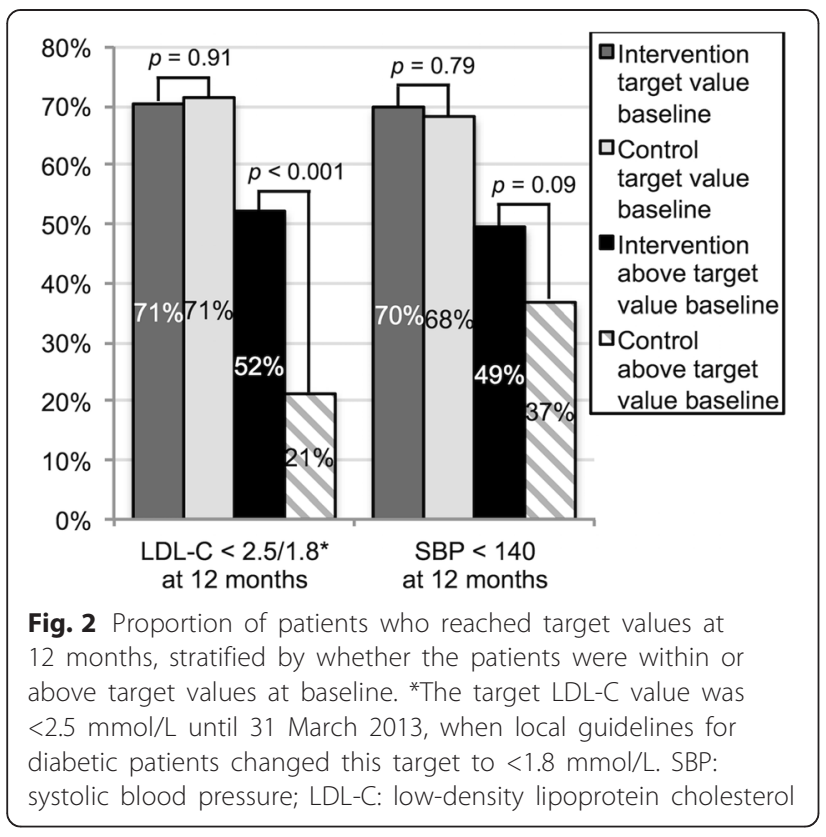

to the proportion of patients reaching target values, the examined intervention was significantly more effective concerning LDL-C, and showed a trend of being more effective for SBP.

Previous population-based studies have shown that approximately $15-60 \%$ of participants reach target values for LDL-C or BP [5-7]. In the present study, as many as $83.8 \%$ of the patients in the intervention group reached the target LDL-C, SBP, and DBP values after medication titration (Fig. 3). At 12 months, the proportion of participants with target values had decreased to $40 \%$. Similarly, a fairly large proportion of the patients with target levels at baseline showed above-target values at 12 months. This might be explained by decreased

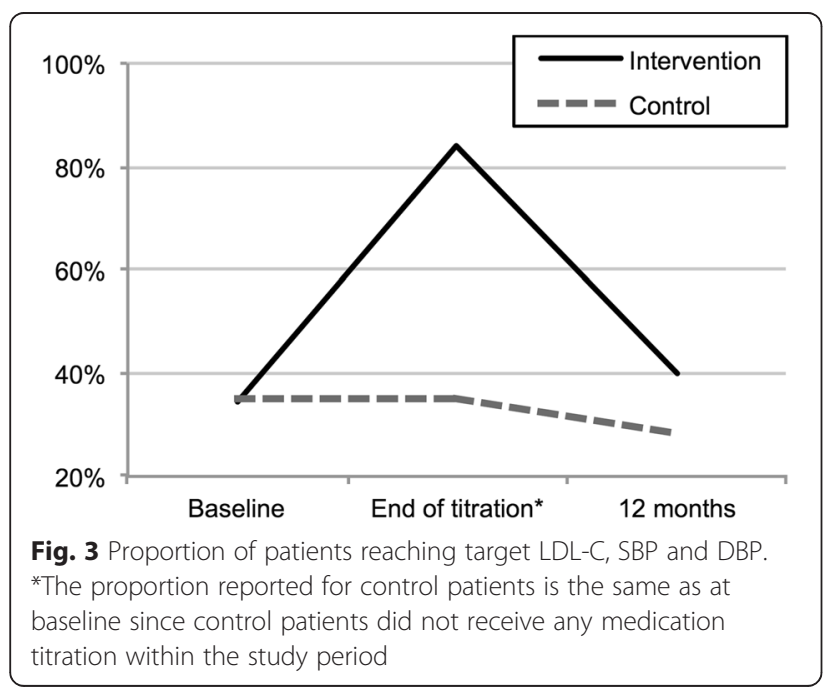

medication adherence over time. More frequent followups during the first year are probably beneficial, but our present findings indicate that secondary prevention efforts should extend beyond 12 months of follow-up. The NAILED trials are planned to follow-up patients for a minimum of 36 months $[17,18]$.

Our results are in agreement with a previous metaanalysis of 11 studies, which found that telehealth interventions (phone and internet communication between patient and healthcare providers) effectively reduced risk factors among patients with coronary heart disease [10]. The meta-analysis showed that intervention decreased the weighted mean difference for LDL-C by $0.41 \mathrm{mmol} /$ $\mathrm{L}$ and for SBP by $4.69 \mathrm{mmHg}$. This can be compared to the presently observed median decrease of $0.8 \mathrm{mmol} / \mathrm{L}$ in LDL-C and of $5 \mathrm{mmHg}$ in SBP. The present study showed a larger risk factor reduction, even though the median patient age was 75 years compared to the mean age of 57-64 years in the meta-analysis [10]. The age of the NAILED study population is more representative of the general ACS, stroke, and TIA population than the younger patients included in previous trials evaluating telephone-supported secondary preventive follow-up.

We believe that prompt medication titration and follow-up every four weeks until target values are reached represent a key component of the NAILED method. Two recently published Cochrane reviews evaluate organizational strategies to improve risk factor control after a CVD, and report only weak effects or no effect on modifiable risk factor levels. None of the included studies that are available in English used a method involving prompt medication titration $[8,9]$. In the PREVENTION study, prompt medication titration performed by pharmacists authorized to prescribe medication resulted in increased proportions of patients reaching the desired risk factor levels [21].

Our separate analyses of DM or CKD patients showed that the CKD patients seemed to gain more from the NAILED intervention. This may be partly explained by the fact that DM patients are carefully monitored within the Swedish healthcare system. In the county of Jamtland, DM patients have a standard yearly visit to the GP and a yearly visit to a nurse for evaluation of the patients' diabetes-associated problems, such as BP and LDL. Consequently, their risk factors are typically better monitored compared to the general CVD population.

The greater benefit seen in the intervention patients with CKD may also be due to the concerns that GPs have regarding medication usage in patients with decreased kidney function, which may make them hesitant to intensify treatment. A meta-analysis investigating statin treatment in CKD patients reported that statins had beneficial effects on mortality and cardiovascular events in predialysis CKD patients, without increased adverse effects 
[22]. Patients with terminal disease were not included in the NAILED trials. Thus, it is unclear why $85.7 \%$ of the control CKD patients who were above target LDL-C levels at baseline were still above target at 12 months.

\section{Limitations}

The method used in the NAILED trial was designed to be broadly implementable in clinical practice and, therefore, all patients were considered for inclusion if they were able to participate and were not enrolled in a concurrent trial. A patient's ability to participate was assessed by a study nurse, introducing a risk of arbitrariness in the decision of eligibility. Another potential weakness was the BP measurement procedure, which was performed by a large number of healthcare professionals at different healthcare centres in the county and using different devices. The letter of referral included simple instructions for what was considered a standardized BP measurement $[17,18]$, but the accuracy of the individual BP measurements could have varied. However, this should not be a major concern, since it is reasonable to believe that any inaccuracy would have been fairly equally distributed between the two study groups. Finally, we defined CKD according to an estimate from a single creatinine value. This differs from the recommended definition of $\mathrm{CKD}$, which requires two measurements taken three months apart [20].

\section{Conclusion}

Our present results showed that nurse-based telephone follow-up including medication titration was more effective than usual care for reducing LDL-C levels and increasing the proportion of patients showing target LDL-C values at 12 months after an ACS, stroke, or TIA among high-risk patients with DM or CKD. The corresponding decrease in SBP and increase in the proportion of patients with target SBP values at 12 months did not significantly differ from those seen in patients with usual care.

The results support our hypothesis that the NAILED follow-up is more efficient than usual care to reduce levels of modifiable risk factors after a CVD in patients with DM or CKD. Future reports will reveal whether nurse-based telephone follow-up is more effective than usual care to achieve long-term risk factor control.

\section{Additional files}

Additional file 1: Risk factor values for patients who had target values at baseline. (PDF $68 \mathrm{~kb}$ )

Additional file 2: Risk factor values for DM patients who had above-target values at baseline. (PDF $75 \mathrm{~kb}$ )

Additional file 3: Risk factor values for CKD patients who had above-target values at baseline. (PDF $70 \mathrm{~kb}$ )

\section{Competing interests}

The authors declare that they have no competing interests.

\section{Authors' contributions}

SJ acquired the data, performed statistical analyses, and drafted the manuscript. TM conceived the study, participated in its design and coordination, helped to draft the manuscript, and revised the manuscript critically for important intellectual content. Al and FB revised the manuscript critically for important intellectual content. All authors contributed to the study design and data interpretation, and all authors read and approved the final manuscript.

\section{Authors' information}

Not applicable.

\section{Acknowledgements}

None.

Received: 28 June 2015 Accepted: 25 September 2015

Published online: 14 October 2015

\section{References}

1. Dhamoon MS, Tai W, Boden-Albala B, Rundek T, Paik MC, Sacco RL, et al. Risk of myocardial infarction or vascular death after first ischemic stroke: the Northern Manhattan study. Stroke. 2007;38(6):1752-8. doi:10.1161/ STROKEAHA.106.480988.

2. SWEDEHEART Annual report 2014. http://www.ucr.uu.se/swedeheart/ index.php/dokument-sh/arsrapporter. 2015.

3. Ulvenstam A, Kajermo U, Modica A, Jernberg T, Soderstrom L, Mooe T. Incidence, trends, and predictors of ischemic stroke 1 year after an acute myocardial infarction. Stroke. 2014;45(11):3263-8. doi:10.1161/ STROKEAHA.114.005770.

4. Perk J, De Backer G, Gohlke H, Graham I, Reiner Z, Verschuren M, et al. European guidelines on cardiovascular disease prevention in clinical practice (version 2012). The fifth joint task force of the European society of cardiology and other societies on cardiovascular disease prevention in clinical practice (constituted by representatives of nine societies and by invited experts). Eur Heart J. 2012;33(13):1635-701. doi:10.1093/eurheartj/ ehs092.

5. Alvarez-Sabin J, Quintana M, Hernandez-Presa MA, Alvarez C, Chaves J, Ribo $M$. Therapeutic interventions and success in risk factor control for secondary prevention of stroke. J Stroke Cerebrovasc Dis. 2009;18(6):460-5. doi:10.1016/j.jstrokecerebrovasdis.2009.01.014.

6. Bhatt DL, Steg PG, Ohman EM, Hirsch AT, Ikeda Y, Mas JL, et al. International prevalence, recognition, and treatment of cardiovascular risk factors in outpatients with atherothrombosis. JAMA. 2006;295(2):180-9. doi:10.1001/ jama.295.2.180.

7. Kotseva K, Wood D, De Bacquer D, De Backer G, Ryden L, Jennings C et al. EUROASPIRE IV: A European Society of Cardiology survey on the lifestyle, risk factor and therapeutic management of coronary patients from 24 European countries. Eur J Prev Cardiol. 2015. doi:10.1177/2047487315569401.

8. Buckley BS, Byrne MC, Smith SM. Service organisation for the secondary prevention of ischaemic heart disease in primary care. Cochrane Database Syst Rev. 2010;3:CD006772. doi:10.1002/14651858.CD006772.pub2.

9. Lager KE, Mistri AK, Khunti K, Haunton VJ, Sett AK, Wilson AD. Interventions for improving modifiable risk factor control in the secondary prevention of stroke. Cochrane Database Syst Rev. 2014;5:CD009103. doi:10.1002/ 14651858.CD009103.pub2.

10. Neubeck L, Redfern J, Fernandez R, Briffa T, Bauman A, Freedman SB. Telehealth interventions for the secondary prevention of coronary heart disease: a systematic review. Eur J Cardiovasc Prev Rehabil. 2009;16(3):281-9. doi:10.1097/HJR.0b013e32832a4e7a.

11. Arnett DK, Goodman RA, Halperin JL, Anderson JL, Parekh AK, Zoghbi WA. AHA/ACC/HHS strategies to enhance application of clinical practice guidelines in patients with cardiovascular disease and comorbid conditions: from the American Heart Association, American College of Cardiology, and US Department of Health and Human Services. Circulation. 2014;130(18):1662-7. doi:10.1161/CIR.0000000000000128.

12. Donahoe SM, Stewart GC, McCabe CH, Mohanavelu S, Murphy SA, Cannon CP, et al. Diabetes and mortality following acute coronary syndromes. JAMA. 2007;298(7):765-75. doi:10.1001/jama.298.7.765.

13. Weiner DE, Tighiouart H, Stark PC, Amin MG, MacLeod B, Griffith JL, et al. Kidney disease as a risk factor for recurrent cardiovascular disease and mortality. Am J Kidney Dis. 2004;44(2):198-206. 
14. Lozano R, Naghavi M, Foreman K, Lim S, Shibuya K, Aboyans V, et al. Global and regional mortality from 235 causes of death for 20 age groups in 1990 and 2010: a systematic analysis for the Global Burden of Disease Study 2010. Lancet. 2012;380(9859):2095-128. doi:10.1016/S0140-6736(12)61728-0.

15. Danaei G, Finucane MM, Lu Y, Singh GM, Cowan MJ, Paciorek CJ, et al.

National, regional, and global trends in fasting plasma glucose and diabetes prevalence since 1980: systematic analysis of health examination surveys and epidemiological studies with 370 country-years and 2.7 million participants. Lancet. 2011;378(9785):31-40. doi:10.1016/S01406736(11)60679-X.

16. Jha V, Garcia-Garcia G, Iseki K, Li Z, Naicker S, Plattner B, et al. Chronic kidney disease: global dimension and perspectives. Lancet. 2013:382(9888):260-72. doi:10.1016/S0140-6736(13)60687-X

17. Mooe T, Bergstrom L, Irewall AL, Ogren J. The NAILED stroke risk factor trial (nurse based age independent intervention to limit evolution of disease after stroke): study protocol for a randomized controlled trial. Trials. 2013;14:5. doi:10.1186/1745-6215-14-5.

18. Mooe T, Bjorklund F, Graipe A, Huber D, Jakobsson S, Kajermo U, et al. The Nurse-Based Age Independent Intervention to Limit Evolution of Disease After Acute Coronary Syndrome (NAILED ACS) risk factor trial: protocol for a randomized controlled trial. JMIR Res Protoc. 2014;3(3):e42. doi:10.2196/ resprot.3466.

19. Levey AS, Stevens LA, Schmid CH, Zhang YL, Castro 3rd AF, Feldman HI, et al. A new equation to estimate glomerular filtration rate. Ann Intern Med. 2009;150(9):604-12.

20. National KF. K/DOQI clinical practice guidelines for chronic kidney disease: evaluation, classification, and stratification. Am J Kidney Dis. 2002:39(2 Suppl 1):S1-266.

21. McAlister FA, Majumdar SR, Padwal RS, Fradette M, Thompson A, Buck B, et al. Case management for blood pressure and lipid level control after minor stroke: PREVENTION randomized controlled trial. CMAJ. 2014;186(8):577-84. doi:10.1503/cmaj.140053.

22. Palmer SC, Craig JC, Navaneethan SD, Tonelli M, Pellegrini F, Strippoli GF. Benefits and harms of statin therapy for persons with chronic kidney disease: a systematic review and meta-analysis. Ann Intern Med. 2012;157(4):263-75. doi:10.7326/0003-4819-157-4-201208210-00007.

\section{Submit your next manuscript to BioMed Central and take full advantage of:}

- Convenient online submission

- Thorough peer review

- No space constraints or color figure charges

- Immediate publication on acceptance

- Inclusion in PubMed, CAS, Scopus and Google Scholar

- Research which is freely available for redistribution 